\title{
Increased Cerebral Cortex Perfusion in Stroke Patients with Cognitive Disorder Following Cilostazol Administration: Two Cases Report
}

\author{
Yukihiro Hara, Shigeru Obayashi
}

Department of Rehabilitation Medicine, Nippon Medical School, Chiba Hokusoh Hospital, Chiba, Japan.

Email: hara-y@nms.ac.jp

Received September $14^{\text {th }}, 2012$; revised October $18^{\text {th }}, 2012$; accepted October $30^{\text {th }}, 2012$

\begin{abstract}
Objective: In our search for a new augmentation therapy for stroke patients, we administered cilostazol, an antiplatelet agent. Subjects: The patients suffered from mild hemiparesis or cognitive disorder showing reduced cerebral perfusion in the prefrontal cortex. Methods: We evaluated the functional cerebral blood flow (CBF) before and after the administration of cilostazol using near-infrared resonance spectroscopy (NIRS) during a verbal fluency task (VFT). Results: For the patient with cognitive disorder, statistically significant improvements were observed in the number of generated words in the VFT before and after administration of $50 \mathrm{mg}$ cilostazol $(\mathrm{p}<0.05$, Mann-Whitney U test). Another patient without cognitive disorder, however, showed no significant VFT improvement after administration of cilostazol. Effect size data revealed large or very large effects of cilostazol on brain activation (oxy-Hb levels) at the affected side prefrontal cortex for both patients. The patient with cognitive disorder showed significant improvement in VFT performance as well as an increase in bilateral prefrontal CBF after cilostazol administration. Discussion: These findings suggest that, for patients with cerebrovascular lesions suffering from cognitive disorder, cilostazol may be promising as a drug to improve cognitive function in addition to preventing recurrent cerebral infarction.
\end{abstract}

Keywords: Stroke; Cognitive Disorder; Brain Perfusion; Antiplatelet Drug; NIRS

\section{Introduction}

Treatment of cerebrovascular patients with cognitive disorder is often difficult because many patients with this condition respond poorly to drugs and rehabilitation. Cilostazol (Pletaal, Otsuka Pharmaceutical Co., Tokyo, Japan) is an inhibitor of platelet aggregation with vasodilating action that has mainly been used for preventing recurrent cerebral infarction [1]. Cilostazol inhibits type III phosphodiesterase; it has been confirmed in platelets and vascular smooth muscle cells [2] that cilostazol inhibits the degradation of cyclic AMP (cAMP) to AMP and increases the intracellular cAMP concentration. Moreover, in the white matter of rats with artificial chronic cerebral ischemia, increases in phosphorylated cAMP response element binding protein (CREB), Bcl-2, and COX-2 expression levels were reported in the cilostazol-treated group compared with the control group [3]. The activation of these intracellular signaling molecules by cilostazol might enhance neural activity, resulting in an increased cerebral blood flow (CBF) [4].

Using near-infrared resonance spectroscopy (NIRS), a recently developed neuroimaging methodology that allows the repetitive examination of cerebral perfusion while performing cognitive tasks, we were able to test the effectiveness of cilostazol in improving cerebral perfusion during a verbal fluency task (VFT). The advantages of NIRS include its noninvasiveness, portability, the natural setting of the examination, low running cost and its sensitivity. Changes in both oxygenated and deoxygenated hemoglobin concentration on NIRS are interpreted to reflect cortical activation, as these changes have been shown to correspond to regional CBF in functional magnetic resonance imaging studies [5]. In the present study, we measured changes in cerebral hemoglobin concentration using multichannel NIRS during a cognitive task, and investigated the relationship between regional cerebral blood volume and cognitive task performance after cilostazol administration.

\section{Subjects and Methods}

\subsection{Subjects}

One subject was a 70-year-old woman and suffered from cerebral infarction in the right internal capsule for about 7 weeks prior to the experiment with the mild left hemi- 
paresis (Subject A). She showed no cognitive and speech disorder and her activity of daily life (ADL) was independent 5 weeks after onset. Another subject was a 58year-old man who had suffered from cerebral infarction for about 6 weeks prior to the experiment (Subject B). His T2-weighted brain MRI showed cortical and subcortical infarction in the insular cortex and precentral gyrus in the right frontal lobe. His impairments consisted of very mild left hemiparesis and cognitive disorders including inattention and visual extinction without speech disturbance. His cognitive function tests were Mini Mental State Examination score; 29/30 and Wechsler Adult Intelligent Scale-3rd edition score; Performance IQ 95, Verbal IQ 119, total IQ 110. His ADL was completely independent 2 weeks after the stroke onset. His 99mTc-ECD single-photon emission computed tomography showed a decrease in $\mathrm{CBF}$ in the affected right prefrontal lobe area. Both of them had no history of other neurological or psychiatric disorders. They had no antiplatelet drugs and the doses of the other drugs used were fixed 4 weeks prior to cilostazol administration.

\subsection{Methods}

To determine whether any distinct brain activities could be detected by NIRS, we measured changes in oxygenated hemoglobin concentration (oxy-Hb), deoxygenated hemoglobin concentration (deoxy-Hb) and total hemoglobin concentration (total- $\mathrm{Hb}$ ) during the use of the phonological version of the VFT using the 22-channel NIRS system (ETG-4000 Optical Topography System; Hitachi Medical Co., Tokyo, Japan). The machine uses two wavelengths of near-infrared light (695 and $830 \mathrm{~nm}$ ) with a temporal resolution of $10 \mathrm{~Hz}$ to measure relative changes of absorbed near-infrared light. The distance between pairs of emission and detector probes was set at $30 \mathrm{~mm}$, which enabled cerebral blood volume measurements at a $2-\mathrm{cm}$ to $3-\mathrm{cm}$ depth from the skin of the head, approximately at the surface of the cerebral cortices [5]. We used a probe set (plastic panel) of optodes. The set consisted of eight light emitters and seven detectors resulting in 22 channels with an area of $12 \times 6 \mathrm{~cm}$ on the scalp. The $3 \times 5$ probes were attached to the subject's medial frontal area, whose long axis was arranged orthogonally to the line connecting inion to nasion. The probes were symmetrically positioned centering on $\mathrm{Fz}$, in accordance with the international 10/20 system for electroencephalogram electrode placement. The rate of data sampling was $0.1 \mathrm{~s}$.

\subsection{Task Procedure}

The subjects performed a verbal fluency task (VFT) consisting of a 5-s pre-task baseline period, a 20 -s task pe- riod, and a 35-s post-task baseline period. Before the NIRS measurements, the subject was instructed to generate as many words as possible in the task period. The initial syllables assigned were changed every 20 -s $(/ \mathrm{a} /$, $/ \mathrm{ka} / \mathrm{and} / \mathrm{sa} /$, respectively) during the 60 -s task period. The initial syllables assigned were different between before and after cilostazol administration. In the pre-task and the post-task baseline periods, the subject was instructed to repeat a train of syllables (" $/ \mathrm{a} /, / \mathrm{i} /, / \mathrm{u} /, / \mathrm{e} /$, /o/"). The subject was seated in a comfortable chair with his eyes closed. The timing of syllable changes was delivered to the subject by the examiners' cue. The subject was asked to avoid body movements such as neck movements, strong biting, and blinking during the NIRS measurements.

\subsection{Experimental Design}

Before cilostazol administration, the subjects performed a VFT consisting of the repetition of five initial syllables during NIRS measurement. They then took a 50-mg cilostazol OD tablet. Ninety minutes after cilostazol administration, when the blood drug concentration of cilostazol reached the maximum [6], the patient performed a second VFT consisting of the repetition of five different syllables during NIRS measurement. The present study was approved by the Institutional Review Board and Ethics committee of Nippon Medical School Chiba Hokusoh Hospital. Written informed consent was obtained from the subjects before the start of the investigation.

\subsection{Data Analysis}

The moving average method was adopted for the analyzed data to remove short-term motion artifacts with a time window of $5 \mathrm{~s}$. At first, we used multiple data analysis with the ETG-4000 operation system v. 1.11 to display grand mean waveforms of $\mathrm{Hb}$ concentration changes. Data were analyzed using the "integral mode". The pre-task baseline was determined as the mean over a 10 -s period just before the task period. The post-task baseline was determined as the mean $5 \mathrm{~s}, 15 \mathrm{~s}$ after the task period, and linear fitting between the pre- and the post-task baselines was applied to data between the two baselines. For data analyses, the task and the post-task periods were divided into five time segments along the time course, the task period into five segments ("task 1" "task 5" segments for the first to the fifth 20 s periods, respectively). Obtained oxy-Hb data were averaged over these five time segments. For analyses of brain activity, with reference to a recent article by Okamoto and colleagues [7], we placed four regions of interest (ROI) as affected and non-affected prefrontal ROI. The measurement points were superimposed on a template image of a 
three-dimensionally reconstructed cerebral cortex in the NIRS software. To evaluate the effect of cilostazol on cortical activation during VFT, we calculated the effect size to adjust the influence of differential pathlength factors among subjects on oxy-Hb levels [8]. The effect size for the effect of cilostazol on oxy-Hb activities (D oxy$\mathbf{H b})$ was calculated as follows: $\mathbf{D} \mathbf{~ o x y}-\mathbf{H b}=(\mathbf{m}$ oxy-Hb2 - m oxy-Hb1)/Std oxy-Hb1, where m oxy-Hb1, m oxy-Hb2 and Std oxy-Hb1 were mean oxy-Hb level during the VFT period before cilostazol administration, mean oxy-Hb level during the VFT period after cilostazol administration and standard deviation of oxy- $\mathrm{Hb} 1$ level during the VFT period before cilostazol administration, respectively.

\section{Results}

\subsection{Task Performance Data}

Performance on the VFT before and after cilostazol administration was showed on the Table 1. Subject A showed some increased generated words after cilostazol administration at each session, but there was no statisticcally significant difference. There was a statistically sig- nificant difference in the number of words at each 5 session generated before and after cilostazol administration in subject $\mathrm{B}(\mathrm{p}<0.05$, Mann-Whitney $\mathrm{U}$ test $)$. Total words count in 5 sessions also increased extremely after cilostazol administration in subject B.

\section{2. oxy-Hb Changes in Prefrontal Cortex}

Using three-dimensional (3-D) NIRS mapping, we demonstrated the representative grand average waveforms of oxy-Hb changes obtained by NIRS during the VFT in subject B (Figure 1). 3-D NIRS mapping demonstrated a 3-D map of oxy-Hb during the VFT on the reconstructed cerebral cortex. During VFT period, oxy-Hb levels increased bilaterally in prefrontal areas and reached the peak approximately $15 \mathrm{~s}$ after starting VFT. The oxy-Hb

Table 1. Verbal fluency task.

\begin{tabular}{cccccc}
\hline & \multicolumn{2}{c}{ Subject A } & \multicolumn{2}{c}{ Subject B } \\
\hline & Before & After & & Before & After \\
\hline Mean words/session & 4.6 & 5.0 & ns & 4.2 & $7.0^{*}$ \\
Total words/5 sessions & 23 & 25 & & 21 & 35 \\
\hline
\end{tabular}

"Mann Whitney U test, $\mathrm{p}<0.05$.
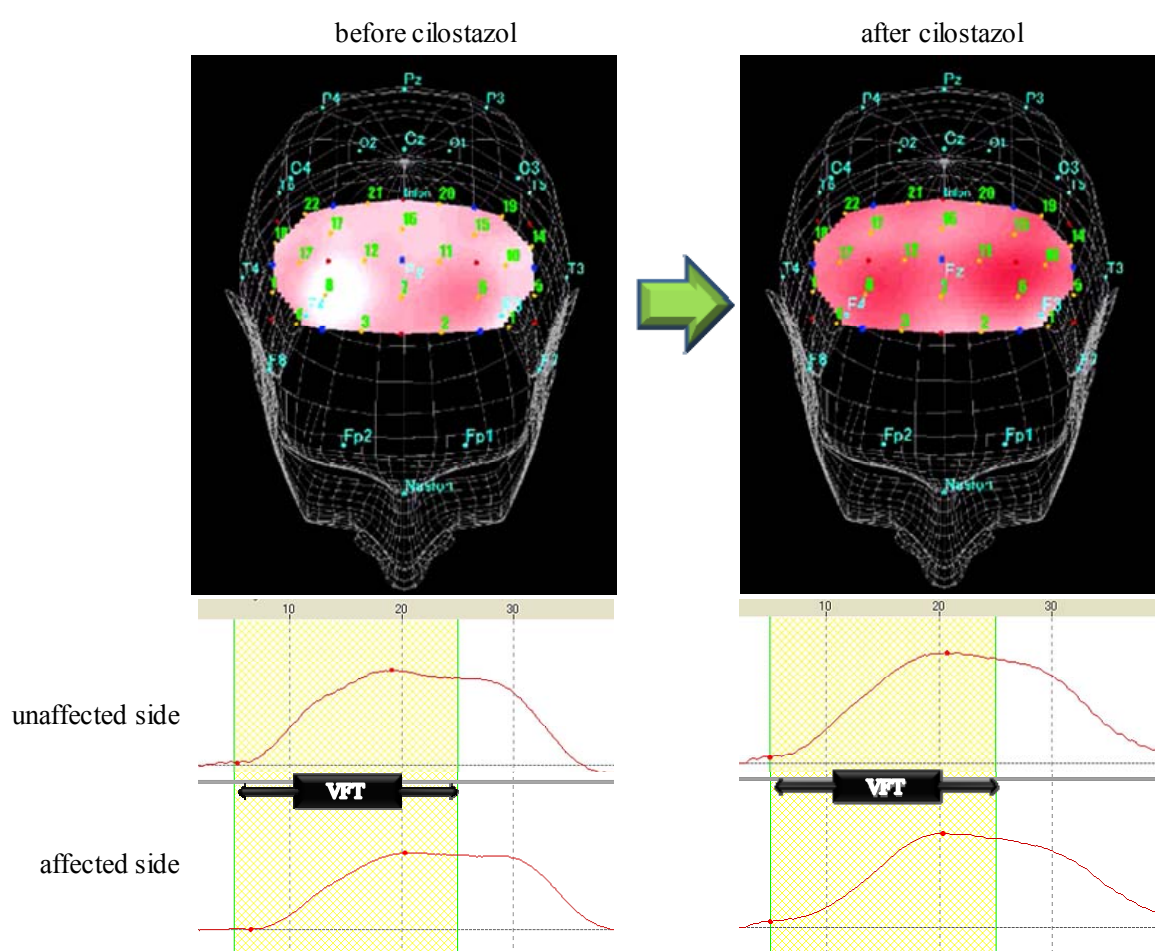

Figure 1. Three-dimensional (3-D) NIRS mapping and grand average waveforms (ROI analysis) in the prefrontal lobe of subject B during a verbal fluency task. Upper) 3-D NIRS mapping image showed poor CBF on the affected side in the prefrontal cortex during the VFT before cilostazol administration. After cilostazol, [oxy-Hb] significantly increased in bilateral, prefrontal lobe during the VFT; Lower) the grand average waveforms of oxy-Hb changes obtained by NIRS during the VFT. 3-D NIRS mapping image and the grand average waveforms showed poor CBF on the affected side in the prefrontal cortex during the VFT before cilostazol administration. After cilostazol administration, [oxy-Hb] significantly increased in bilateral, prefrontal lobe during the VFT. 
before cilostazol mapping image showed poor CBF on the affected side in the prefrontal cortex during the VFT. After cilostazol, the 3-D map of oxy-Hb showed a significant increase in bilateral, prefrontal CBF during the VFT. The mean oxy-Hb activation values during VFT of the affected and unaffected prefrontal ROI before and after cilostazol administration were shown in Table. The effect of preparation on the increase of oxy-Hb levels during the VFT was evaluated by calculating effect sizes (Table 2). The effect size calculated by using oxy-Hb levels showed very large cilostazol effect $(1.10 \leq \mathrm{d}: 1.265$ $<1.45$ ) on affected side and negligible cilostazol effect $(-0.15 \leq \mathrm{d}: 0.046<0.15)$ on unaffected side premotor cortex perfusion in Subject A. The effect size of oxy-Hb levels showed large cilostazol effect $(0.75 \leq \mathrm{d}: 0.923<$ $1.10)$ on affected side and medium cilostazol effect $(0.4$ $\leq \mathrm{d}: 0.437<0.75)$ on unaffected side prefrontal cortex in Subject B. These indicated that activations of the affected side prefrontal cortex area were enhanced by cilostazol during VFT.

\section{Discussion}

We reported the cases of patient who had suffered a stroke with or without cognitive impairment and prefrontal lobe hypoperfusion. The normal 22 elder control subjects produced a mean 21.91 words (S.D. 5.38) in a total of 5 task VFT sessions (our other study data). Subject A without cognitive impairment showed same VFT result as them with little improvement after cilostazol administration. The administration of a single $50-\mathrm{mg}$ dose of cilostazol immediately improved VFT performance and prefrontal cerebral perfusion on both the affected and unaffected hemispheres in subject B with cognitive impairment. In addition, he showed improvements in cognitive disorder symptoms as well as an increase in cerebral blood flow during the VFT revealed by NIRS. The oxy-Hb increases in NIRS during the VFT were smaller in the affected than in the unaffected side before cilostazol administration. The statistically significant main effects on oxy-Hb increase were obtained after the administration of $50 \mathrm{mg}$ cilostazol in the affected hemisphere in the prefrontal cortex. The methods used in the present study are well established for analysis of baseline vs. treatment comparisons. VFT is one measure of frontal lobe cognitive function, and its validity and test-retest reliability have been examined [9]. Significant correlations were observed between the VFT and digit

Table 2. NIRS effect size.

\begin{tabular}{ccc}
\hline & Subject A & Subject B \\
\hline Affected side & 1.265 & 0.923 \\
Unaffected side & 0.046 & 0.437 \\
\hline
\end{tabular}

cancellation tasks, Stroop tasks and sentence recall. Because the initial syllables assigned differed between the two experiments, there could be very little possibility of a learning effect in the present experimental VFT protocol [9]. Using a 24-channel NIRS in 12 healthy men, Kono et al. [10] examined the replicability of oxy- $\mathrm{Hb}$ and deoxy-Hb concentration changes in the prefrontal cortex during VFT. Most studies of the effects of cilostazol on cerebral perfusion report the long-term effects of cilostazol in static conditions. It has been reported that longitudinal administration of cilostazol improves CBF in patients with chronic cerebral infarction and improves cerebral function evaluated by P300 event-related potential [11], and the enhancing effect in CBF may have led to improvements of depressive symptoms in post-stroke depression [11]. It may be postulated that increased CBF due to cilostazol results in the activation of intracellular signaling molecules enhancing neural activity through the neuron-capillary coupling [4]. Alternative explanations for increased cerebral perfusion include the presservation of endothelium-dependent vasodilation through cilostazol-induced endothelial protective effects [12]. The present study is the first report showing the short-term augmentation effects of cilostazol on prefrontal cerebral perfusion during cognitive tasks in stroke patients. The beneficial effects of cilostazol on CBF could be attributeable to rheological improvement caused by its vasodilating action and to its inhibition of platelet aggregation. As in the cases presented here, cognitively impaired patients with cerebrovascular lesions often respond poorly to drugs and develop adverse reactions, and the symptoms of cognitive impairment tend to persist and show little improvement despite cognitive rehabilitation. Our findings, however, suggest that one dose of $50 \mathrm{mg}$ cilostazol, half of the one-time daily dose, may be effective in augmenttation therapy for stroke patients suffering from cognitive disorders and promote the stroke patients rehabilitation. In future, we will investigate the long-term effects of cilostazol on the cognitive function as well as prefrontal cerebral perfusion. The authors declare that there is no financial or other conflict of interest for this study.

\section{REFERENCES}

[1] Y. Shinohara, Y. Katayama, S. Uchiyama, T. Yamaguchi, S. Handa, K. Matsuoka, Y. Ohashi, N. Tanahashi, H. Yamamoto, C. Genka, Y. Kitagawa, H. Kusuoka, K. Nishimaru, M. Tsushima, Y. Koretsune, T. Sawada and C. Hamada, "Cilostazol for Prevention of Secondary Stroke (CSPS 2): An Aspirin-Controlled, Double-Blind, Randomised Non-Inferiority Trial," Lancet Neurology, Vol. 9, No. 10, 2010, pp. 959-968. doi:10.1016/S1474-4422(10)70198-8

[2] T. Tanaka, T. Ishikawa, M. Hagiwara, K. Onoda, H. Itoh 
and H. Hidaka, "Effects of Cilostazol, a Selective CAMP Phosphodiesterase Inhibitor on the Contraction of Vascular Smooth Muscle," Pharmacology, Vol. 36, No. 5, 1988, pp. 313-320. doi.org/10.1159/000138400

[3] T. Watanabe, N. Zhang, M. Liu, R. Tanaka, Y. Mizuno and T. Urabe, "Cilostazol Protects Against Brain White Matter Damage and Cognitive Impairment in a Rat Model of Chronic Cerebral Hypoperfusion," Stroke, Vol. 37, No. 6, 2006, pp. 1539-1545. doi:10.1161/01.STR.0000221783.08037.a9

[4] S. Kobayashi, S.Yamaguchi, T. Katsube, M. Kitani, K. Okada and T. Tsunematsu, "Long-Term Effect of Cilostazol on Cerebral Blood Flow in Chronic Cerebral Infarction," Arzneimittelforshung, Vol. 35, No. 7A, 1985, pp. 1193-1197.

[5] V. Toronov, A. Webb, J. H. Choi, M. Wolf, A. Michalos, E. Gratton and D. Hueber, "Investigation of Human Brain Hemodynamics by Simultaneous Near-Infrared Spectroscopy and Functional Magnetic Resonance Imaging," Medical Physics, Vol. 28, No. 4, 2001, pp. 521-527. doi:10.1118/1.1354627

[6] H. Akiyama, S. Kudo and T. Shimizu, "The Absorption, Distribution and Excretion of a New Antithrombotic and Vasodilating Agent, Cilostazol, in Rat, Rabbit, Dog and Man," Arzneimittelforschung, Vol. 35, No. 7A, 1985, pp. 1124-1132.

[7] M. Okamoto, H. Dan, K. Sakamoto, K. Takeo, K. Shimizu, S. Kohno, I. Oda, S. Isobe, T. Suzuki, K. Kohyama and I. Dan, "Three-Dimensional Probabilistic Anatomical Cranio-Cerebral Correlation via the International 10-20 System Oriented for Transcranial Functional Brain Map-

\section{List of Abbreviations}

CBF: cerebral blood flow.

CAMP: cyclic AMP.

NIRS: near-infrared resonance spectroscopy.

VFT: verbal fluency task.

ADL: activity of daily life.

oxy-Hb: oxygenated hemoglobin concentration.

deoxy-Hb: deoxygenated hemoglobin concentration.

total-Hb: total hemoglobin concentration.

3-D: three-dimensional. ping," Neuroimage, Vol. 21, No. 1, 2004, pp. 99-111. doi:10.1016/j.neuroimage.2003.08.026

[8] M. L. Schroeter, S. Zysset, F. Kruggel and D. Y. von Cramon, "Age Dependency of the Hemodynamic Response as Measured by Functional Near-Infrared Spectroscopy," Neuroimage, Vol. 19, No. 3, 2003, pp. 555-564. doi:10.1016/S1053-8119(03)00155-1

[9] J. E. Harrison, P. Buxton, M. Husain and R. Wise, "Short Test of Semantic and Phonological Fluency: Normal Performance, Validity and Test-Retest Reliability," British Journal of Clinical Psychiatry, Vol. 39, No. 2, 2000, pp. 181-191. doi:10.1348/014466500163202

[10] T. Kono, K. Matsuo, K. Tsunashima, K. Kasai, R. Takizawa, M. A. Rogers, H. Yamasue, T. Yano, Y. Taketani and N. Kato, "Multiple-Time Replicability of Near-Infrared Spectroscopy Recording during Prefrontal Activation Task in Healthy Men," Neuroscience Research, Vol. 57, No. 4, 2007, pp. 504-512. doi:10.1016/j.neures.2006.12.007

[11] Y. Mochizuki, M. Oishi and T. Mizutani, "Effects of Cilostazol on Cerebral Blood Flow, P300, and Serum Lipid Levels in the Chronic Stage of Cerebral Infarction," Journal of Stroke and Cerebrovascular Disease, Vol. 10, No. 2, 2001, pp. 63-69. doi:10.1053/jscd.2001.24657

[12] T. Nakamura, H. Houchi, A. Minami, S. Sakamoto, K. Tsuchiya, Y. Niwa, K. Minakuchi and Y. Nakaya, "Endotheliumdependent Relaxation by Cilostazol, a Phosphodiesterase III Inhibitor, on Rat Thoracic Aorta," Life Science, Vol. 69, No. 15, 2001, pp. 1709-1715. doi:10.1016/S0024-3205(01)01258-9

ROI: regions of interest.

D oxy-Hb: effect size for the effect of cilostazol on oxy-Hb activities.

m oxy-Hb1: mean oxy-Hb level during the VFT period before cilostazol administration.

m oxy-Hb2: mean oxy-Hb level during the VFT period after cilostazol administration.

Std oxy-Hb1: standard deviation of oxy-Hb1 level during the VFT period before cilostazol administration. 